\title{
The Impact of Footwear on Posture, Gait and Balance
}

\author{
Saori Karasawa ${ }^{1,2}$, Munetaka Yamamoto ${ }^{1,2}$, Junko Sakurai ${ }^{3}$, Shiori Kawasaki ${ }^{1,2}$, \\ Hiroyuki Kobayashi1,2
}

${ }^{1}$ Department of Hospital Administration, Juntendo Graduate School of Medicine, Tokyo, Japan

${ }^{2}$ Patient Safety Management Section, Juntendo University Hospital, Tokyo, Japan

${ }^{3}$ Faculty of Health and Nursing, Juntendo University, Chiba, Japan

Email: skarasa@juntendo.ac.jp

How to cite this paper: Karasawa, S., Yamamoto, M., Sakurai, J., Kawasaki, S. and Kobayashi, H. (2022) The Impact of Footwear on Posture, Gait and Balance. Health, 14, 209-218.

https://doi.org/10.4236/health.2022.142016

Received: January 4, 2022

Accepted: February 20, 2022

Published: February 23, 2022

Copyright $\odot 2022$ by author(s) and Scientific Research Publishing Inc. This work is licensed under the Creative Commons Attribution International License (CC BY 4.0).

http://creativecommons.org/licenses/by/4.0/

\section{(c) (i) Open Access}

\begin{abstract}
Background: The objective of this study was to investigate the effects of footwear on posture and balance while walking. The types of footwear investigated in this study were open back shoes, commonly worn by hospitalized patients, and closed back shoes. Previous studies have shown that open back shoes, or slippers, increase the risk of falling (among elderly). We hypothesized that our findings would suggest that open back shoes negatively affect gait mechanics in healthy individuals. Methods: Healthy individuals $(\mathrm{n}=12)$ participated in a walking test while wearing closed back shoes and open back shoes. The explanatory variables in this study were the analysis of gait, posture, and balance before and after walking. The objective variable was footwear (closed back shoes vs. open back shoes). A paired t-test was performed to detect significant differences between the two conditions. Results: Among the test items measured, we found a significant difference in minimum wide tilt angle and leftright differences in step length and intensity while walking between the conditions of closed back shoes and open back shoes. These results suggest that open back shoes could negatively impact posture and balance while walking, even in healthy subjects. Conclusion: It is imperative to improve patient awareness of the risk of falling. We believe that the inclusion of our findings in educational pamphlets and in-house notices could help improve patient awareness and more effectively prevent falls among patients.
\end{abstract}

\section{Keywords}

Footwear, Posture, Walking Test, Health, Fall

\section{Introduction}

At Juntendo University Hospital, various measures are taken to prevent patients 
from falling, including utilizing fall risk assessment scores, color coding patients with silicon wristbands, and distributing educational brochures such as "moving safely using a cane".

Despite these efforts to prevent falls, more than 300 falls are reported annually at Juntendo University Hospital. Falls can result in serious injuries and fractures. Even if the injury is non-life-threatening, patient quality of life can be severely affected as a consequence of prolonged hospital stays, higher medical costs, and restricted physical activity [1] [2] [3] [4].

Previous studies have reported that muscle weakness compromises gait kinetics and predisposes a person to falls [4] [5]. It has been speculated that prolonged bed rest and immobilization from extended hospital stays can decrease muscle strength [6], leading to changes in balance and posture during in-hospital ambulation. The risk of falling is also higher for elderly patients who are hospitalized compared to seniors who live at home. Elderly patients tend to lose muscle strength and bone mass in their lower extremities more quickly due to medical treatments and environmental changes have undergone while hospitalized.

As described above, there are complex internal factors that elevate the risk of accidental falls, including age, the total duration of hospital stays, and underlying medical conditions.

Various external factors are known to increase the risk of falling among patients, including the difference in heights of surfaces while stepping, inadequate lighting, and unfamiliar environments. In this study, we focused on the external factor, "footwear". Research regarding footwear often involves investigating the sole of the shoe. Data from past research [7] suggests that the harder the sole, the larger the contact area of the sole, and the lower the heel, the more stable walking becomes. However, there are few scientific studies that investigate the effects of open back shoes, or slippers, on gait. We believe that one of the reasons there are so few studies investigating the use of open back shoes is because the use of indoor slippers and hospital slippers is not very common outside of Japan. Open back shoes, such as slippers and sandals, have been associated with a higher risk of falling due to poor grip abilities and its tendency to cause the wearer to slip and trip. In particular, slippers are often made of slippery material and because there is no back to the shoe, it can cause loss of balance, and therefore, unstable walking [8]. In fact, some hospitals have successfully reduced in-hospital falls by recommending closed back shoes to patients [9]. At Juntendo University Hospital, we recommend wearing closed back shoes instead of open back shoes to our patients for inpatient footwear. Because of this, the amount of falls due to footwear has been decreasing. However, while fall incidents have been decreasing, it hasn't been eliminated because some patients still prefer to wear open back shoes. Even though we have a culture of wearing open back shoes in Japan, there are a lot of retrospective reviews, such as fall incident analysis and questionnaire surveys regarding slipper use.

Accordingly, we surmised that footwear could negatively affect gait parame- 
ters, balance, and posture, not only in hospitalized patients but also in healthy individuals.

In this study, we analyzed the characteristics of balance and posture control with a wearable sensor on healthy individuals while wearing closed back shoes and open back shoes during a walking test and compared the results. By scientifically analyzing fall risks associated with footwear as reported in earlier studies, and by demonstrating the negative impact of open back shoes on gait parameters, we aim to raise the awareness of risks associated with in-hospital falls.

\section{Materials and Methods}

\subsection{Subjects and Sample Size}

A total of 12 faculty and medical staff members of Juntendo University Hospital were randomly recruited as subjects ( 6 male and 6 females, mean age 38.6 years, range 20 - 60 years) to participate in a walking test (Table 1). Participants were deemed eligible if there was no age-related decline in daily walking ability and if they did not have any underlying medical conditions and/or physical disability, particularly of the lower extremities, as this could significantly impact gait. All of the subjects were informed about the possible risks and discomforts that may occur during the experiment prior to receiving their written informed consent to voluntarily participate in the study.

Table 1. Background of the subjects.

\begin{tabular}{cccccc}
\hline$\#$ & gender & $\begin{array}{c}\text { Age } \\
(\text { years })\end{array}$ & $\begin{array}{c}\text { Height } \\
(\mathrm{cm})\end{array}$ & $\begin{array}{c}\text { Weight } \\
(\mathrm{kg})\end{array}$ & $\begin{array}{c}\text { BMI } \\
\left(\mathrm{kg} / \mathrm{m}^{2}\right)\end{array}$ \\
\hline 01 & M & 46 & 172.4 & 68.9 & 23.2 \\
02 & $\mathrm{M}$ & 36 & 173.6 & 73.3 & 24.3 \\
03 & $\mathrm{~F}$ & 39 & 161.5 & 53.0 & 20.3 \\
04 & $\mathrm{M}$ & 30 & 163.0 & 57.0 & 21.5 \\
05 & $\mathrm{M}$ & 26 & 171.0 & 68.0 & 23.3 \\
06 & $\mathrm{~F}$ & 63 & 149.7 & 57.5 & 25.7 \\
07 & $\mathrm{~F}$ & 34 & 158.0 & 48.0 & 19.2 \\
08 & $\mathrm{~F}$ & 21 & 153.6 & 46.2 & 19.6 \\
09 & $\mathrm{M}$ & 58 & 170.2 & 66.3 & 22.9 \\
10 & $\mathrm{~F}$ & 39 & 162.4 & 45.6 & 17.3 \\
11 & $\mathrm{M}$ & 42 & 176.5 & 79.8 & 25.6 \\
12 & $\mathrm{~F}$ & 29 & 153.0 & 43.6 & 18.6 \\
Mean \pm S.D. & & $\mathbf{3 8 . 6}$ & & & \\
\hline
\end{tabular}




\subsection{Procedures}

The walking test was performed in the corridor of the Department of Hospital Administration at Juntendo University Graduate School of Medicine. Participants walked 10 meters with closed back shoes or open back shoes. The closed back shoes utilized in this study were shoes the participants themselves wore on daily basis. The open back shoes were slippers that were previously worn and distributed by our hospital. Participants wore wearable biosensor (M-BIT, BITAS Corporation, Tokyo, JAPAN) on their chest to evaluate gait and posture.

\subsection{Wearable Biosensor Device Measurement Method}

According to the sensor's accelerometer measurement manual, the procedure was performed as follows.

1) Using a specialized belt, place the sensor in the middle of the torso.

2) Place the biosensor by yourself or ask for assistance.

3) Stand in an upright position and when ready, press the (Start) button to start the measurement process (the LED lamp will blink green).

4) Stand still for 30 seconds.

5) Starting from the right foot, walk straight for 10 meters.

6) Stop and stand still for 30 seconds.

7) Press and hold the button until the LED lamp turns off to stop measuring.

8) Remove the biosensor from the body.

9) Connect the biosensor to a PC, launch the software for data analysis, and extract results.

Data obtained from analyzing the results of biosensor items:

- Resting postural tilt angle before and after walking (deg);

- Left-right resting postural tilt angle before and after walking (deg);

- Minimum wide tilt angle (deg);

- Step length (sec) during walking: Mean, left, right;

- Step intensity (G) during walking: Mean, left, right;

- Average walking speed $(\mathrm{m} / \mathrm{sec})$;

- Harmonics ratio.

10) After the data is transferred, delete data from the biosensor.

Out of the extracted items, the following were analyzed:

1) Differences in resting postural tilt angle before and after walking (deg);

2) Differences between left-right resting postural tilt angle before and after walking (deg);

3) Minimum wide tilt angle (deg);

4) Left-right differences in step length during walking (sec);

5) Left-right differences in step intensity during walking (G);

6) Average walking speed $(\mathrm{m} / \mathrm{sec})$;

7) Harmonics ratio.

"Tilt angle" refers to anterior and posterior pelvic tilt. The larger the difference between the anterior and posterior pelvic tilt, the larger the tilt. Although 
there are some errors depending on body shape and sensor position, 90 degrees or more is considered a general tilt angle. A tilt angle of 90 degrees or less is considered to be at an angle that's easy to fall while walking [10].

"Left/right angle" refers to lateral left and right pelvic tilt. The larger the difference between the left and right pelvic tilt, the larger the tilt. Similar to "tilt angle", although there are some errors depending on body shape and sensor position, posture is considered good when it's close to 0 degrees.

"Wide tilt angle" is an index to evaluate the overall postural tilt of the body while walking. (Tilt angle and left/right angle combined). The base measurement for "wide tilt angle" is 90 degrees, measured from the ground. The larger the difference from 90 degrees, the worse posture is considered.

"Harmonics ratio" measure smoothness of trunk motion during gait; higher ratios indicate greater smoothness [11].

"Step length" refers to the amount of seconds to take one step. The length and strength of each step are used as an index to evaluate the amount of weight balance of each step. The larger the difference between left and right steps, the worse weight balance is considered.

We also interviewed the participants and recorded personal information, such as age, sex, height, and weight, in which the software prompts for data extraction.

\subsection{Statistical Analysis}

Using footwear (closed back vs. open back) as objective variables, and test items extracted from the biosensors as explanatory variables, we compared the variables using a paired t-test.

For the paired t-test, closed back footwear was designated as the "shoes group" and open back footwear as the "slippers group".

All data is expressed as means \pm standard errors, unless otherwise specified.

\subsection{Ethical Considerations}

This study's design was approved by the Medical Corp Koyokai, Tokyo, Japan (Protocol \# MCK-2019-01). This study was conducted in accordance with the "Ethical Guidelines for Medical Sciences Research for Humans". To protect the privacy and confidentiality of the study subjects, each subject was assigned a unique code number and special attention was paid to data management.

\section{Results}

A paired t-test was performed to determine the association between objective variables (the shoes group vs. the slippers group) and explanatory variables (biosensor data obtained from the 12 subjects). Our results are summarized below as well as in Figure 1.

There were significant differences found in 3 test items between the shoe group and the slippers group. 

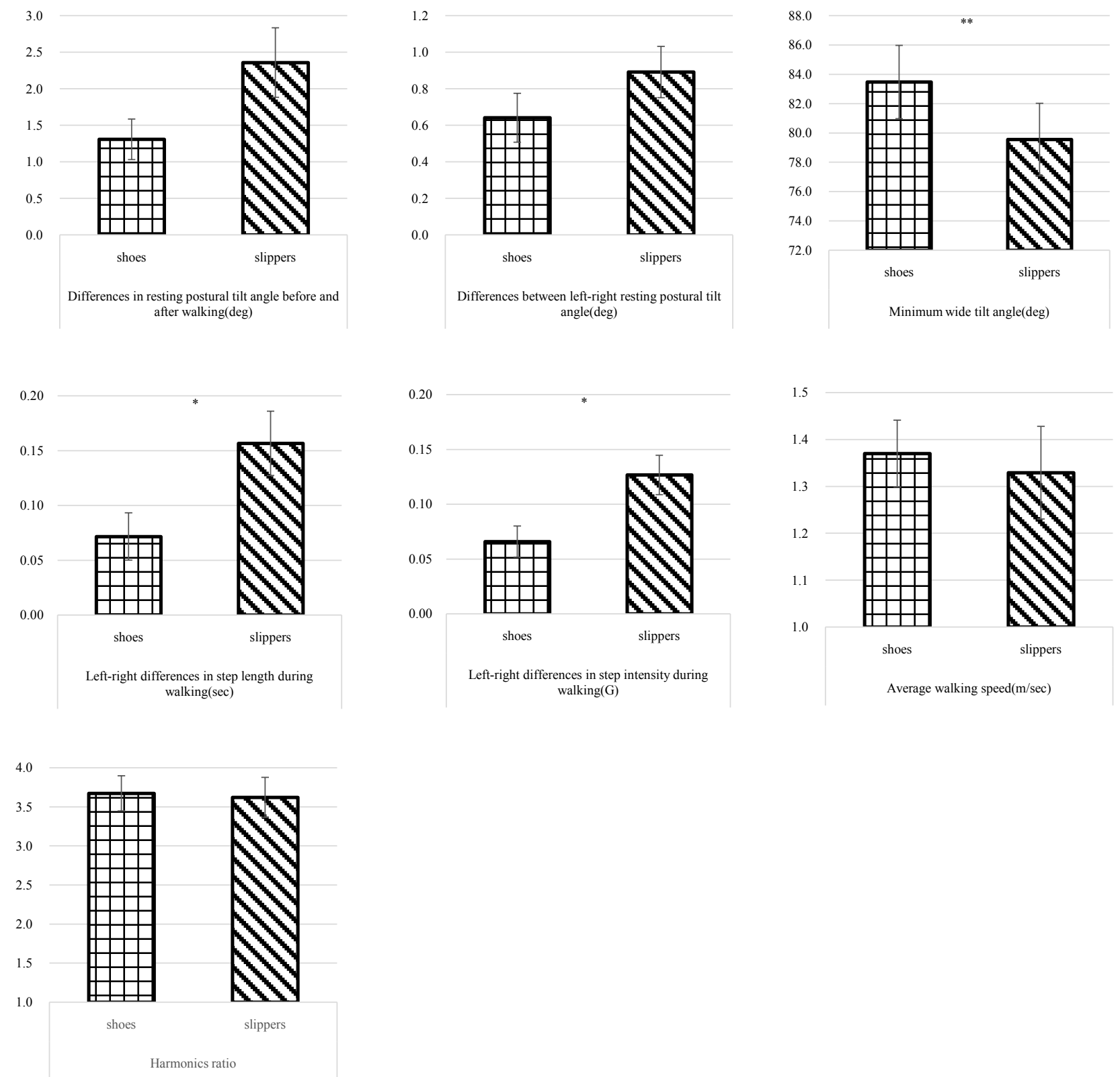

Figure 1. Comparison of walk test results between closed back shoes (shoes) and open back shoes (slippers). Results are expressed as mean \pm S.E, ${ }^{*} \mathrm{p}<0.05,{ }^{*} \mathrm{p}<0.01, \mathrm{n}=12$.

- Minimum wide tilt angle $(\mathrm{p}<0.01)$

The slippers group $(79.55 \pm 2.47)$ had a larger tilt angle than the shoes group (83.48 \pm 2.5$)$.

- Left-right differences in step length during walking $(\mathrm{p}<0.05)$

The slippers group $(0.16 \pm 0.03)$ had a larger difference in left-right step length during walking than the shoes group $(0.07 \pm 0.02)$.

- Left-right differences in step intensity during walking $(\mathrm{p}<0.05)$

The slippers group $(0.13 \pm 0.02)$ had a larger difference in left-right step intensity during walking than the shoes group $(0.07 \pm 0.01)$.

We saw no significant differences in the other 4 test items (differences in resting 
postural tilt angle before and after walking, differences between left-right resting postural tilt angle before and after walking, average walking speed, harmonics ratio) between the shoes group and the slippers group.

Although our small sample size limits statistical validity, our results show preliminary evidence of footwear affecting posture and gait balance in healthy individuals.

\section{Discussion}

Our results showed the impact of footwear on posture and balance while walking. To our knowledge, there have been very few studies until now that provide objective data about this topic.

We found that the slipper group had a significantly smaller minimum wide tilt angle compared to the shoe group. These results suggest that open back shoes negatively impact posture while walking in comparison to closed back shoes. Previous studies on the elderly have shown that a forward leaning posture increases the risk of falling [12] [13] [14]. In our study, a significantly smaller minimum wide tilt angle was found in the slipper condition group compared to the shoe condition group, suggesting that slippers may affect posture while walking and increase the risk of falling.

In addition, the slipper group had significantly higher left-right differences in step length and intensity while walking compared to the shoe group. We conjecture that open back shoes are more likely to disturb the movements of both legs while walking. In this study, the participants in the shoes group wore closed back shoes that they are familiar with, whereas the slippers group used unused open back shoes. Our results suggest that the difference in degree of wear of the sole of the shoe is a factor of walking asymmetry. Increased gait asymmetry generally reflects poor coordination which disturbs gait stability, ultimately increasing the risk of falling [15].

There is an abundance of research studying gait stability among older adults [16]. In this study, we were able to show variance in walking stability due to differences in footwear (closed back shoes vs. open back shoes) in healthy adults. Most falls occur without awareness [17]. Although the reliability of our results could have been compromised by whether or not the subjects had an increased sense of awareness during the test, the testing environment was the same for both types of footwear. In addition, a $10-\mathrm{m}$ walk test is a common method among researchers and is recommended as standard protocol due to the fact that this test can be performed quickly and easily without special equipment [18].

For these reasons, we believe our results to be accurate.

We saw no significant differences in resting posture. A previous study has reported that upright posture is relatively stable when wearing open back shoes because the soles are flat, so our results support their findings [19]. Although no significant difference in average walking speed was found in this study, other research suggests that asymmetry during walking, in addition to walking speed has a great influence on fall risk while walking. Previous studies have shown that the 
slower the walking speed, the higher the risk of falling [20]. This study's results also show that participants in the slipper group walked at a slower pace, which we believe may have contributed to the increased risk of falling while wearing open back shoes. We plan to further investigate this relationship with a large sample group in future studies.

Hospitalized patients usually put on open back shoes to go somewhere, such as to the bathroom or to examination rooms, not just for standing in place. Thus, we believe that patients should be educated about the risks of wearing open back shoes in a hospital environment, even during limited in-hospital ambulation, because suboptimal footwear can compromise posture and balance. We plan to use the results of this study to educate medical professionals, such as nurses, physical therapists, and occupational therapists, as well as healthcare students to accurately observe the walking condition of patients.

Further, earlier studies have demonstrated that gait disturbance may increase the risk of falling, but few hospitals explain these risks to patients with the use of clear and persuasive statements backed by evidence [21]. Juntendo University Hospital recommends wearing closed back shoes instead of open back shoes to all hospitalized patients, but some still prefer to wear slip-ons due to their ease of use. Our findings indicate that open back shoes can negatively affect gait mechanics. We have therefore decided to share our findings in pamphlets and in-house bulletins starting from September 2021. We compared the number of inpatient incidents related to falls and footwear that occurred for 3 months since the release of the new pamphlet in September, with the same months of the previous year. Although the causal relationship of the pamphlet can't be confirmed, the number of fall incidents due to footwear decreased from 6 to 1 . Rather than informing patients individually, we aim to reach a greater audience and contribute to improving patient awareness about the risk of falling through these pamphlets and in-house bulletins.

Current monitoring of falls by healthcare professionals alone is inadequate in preventing accidental falls. Given that the number of hospitalized patients with a high risk of falling is expected to increase with age, it is important to enhance awareness of these risks among patients.

The walking test conducted in this study is very simple and non-invasive, thus, we expect patients to find this test easy to understand and to perform.

A number of studies in Japan and overseas have reported that falls can be reduced by educating patients and their family members about fall prevention [22]. For our next study, we plan to perform a correlational analysis between objective data from our walking test and subjective data from fall risk assessments conducted by nurses who work with patients in hospitalized settings to further explore the feasibility of our experimental method and its potential impact on patient awareness about the risks of falling.

\section{Conclusions}

Our findings suggest that open back footwear can disrupt posture and balance 
while walking, and that the risk of falling may be higher among hospitalized patients wearing slip-on footwear compared to those wearing closed back footwear such as sneakers.

We hope that our findings will contribute to the development and practical applications of effective fall risk assessments in the future.

\section{Acknowledgements}

We would like to express our sincere gratitude to Dr. Takuji Yamaguchi and Ms. Ailing $\mathrm{Hu}$ at Center of Advanced Kampo Medicine and Clinical Research, Juntendo University Graduate School of Medicine, for their guidance and cooperation in conducting this study.

\section{Conflicts of Interest}

The authors declare no conflicts of interest regarding the publication of this paper.

\section{References}

[1] Takashima, M., Yamada, R., Sato, Y., Ito, W., Kondo, K., Okada, K. and Asanuma, Y. (2012) The Actual Circumstances Surrounding Bone Fractures Due to Falls at a University Hospital. The Journal of Japan Society for Health Care Management, 13, 62-64.

[2] Suzuki, T. (2003) Epidemiology, and Implications of Falling among the Elderly. Journal of the Japan Geriatrics Society, 40, 85-94. https://doi.org/10.3143/geriatrics.40.85

[3] Kobayashi, K. and Hiiragi, Y. (2016) Interconnectedness of Falls and Relevant Factors: Examination by Structural Equation Modeling. Sogo Rehabilitation, 44, 59-62.

[4] Egami, K., Hirose, M., Takemura, T., Okamoto, K., Tsuda, Y., Ohama, K., et al. (2011) Extra Medical Costs Due to Falls by Using Incident Reporting and Administrative Profiling Data at a Teaching Hospital in Japan: A Retrospective Case Study. Journal of the Japan Society for Healthcare Administration, 48, 157-169.

[5] Kim, H. (2014) Relating of Fall Risk and Walking (in Japanese). Journal of the Society of Biomechanisms, 38, 233-239. https://doi.org/10.3951/sobim.38.233

[6] Tadenuma, T., Sakai, Y. and Maniwa, S. (2006) Analysis and Countermeasures of Fall Risk Factors in Inpatients (in Japanese). The Journal of the Shimane Medical Association, 26, 264-269.

[7] Aboutorabi, A., Bahramizadeh, M., Arazpour, M., Fadayevatan, R., Farahmand, F., Curran, S. and Hutchins, S.W. (2015) A Systematic Review of the Effect of Foot Orthoses and Shoe Characteristics on Balance in Healthy Older Subjects, Prosthetics and Orthotics International, 40, 170-181. https://doi.org/10.1177/0309364615588342

[8] Watanabe, M. (2015) Footwear to Prevent Falls in Hospitals (in Japanese). Japanese Journal of Fall Prevention, 2, 92.

[9] Matsuda, M., Nakao, M., Masuda, A. and Yamada, Y. (2018) Efforts to Reduce the Number of Falls by Implementation of Footwear Survey (in Japanese). The Journal of Japan Society for Health Care Management, 19, 272 p.

[10] Ko, Y.-C., Ryew, C.-C. and Hyun, S.-H. (2017) Relationship among the Variables of 
Kinematic and Tilt Angle of Whole Body According to the Foot Trip during Gait. Journal of Exercise Rehabilitation, 13, 117-121. https://doi.org/10.12965/jer.1732846.423

[11] Lowry, K.A., Lokenvitz, N., and Smiley-Oyen, A.L. (2012) Age- and Speed-Related Differences in Harmonic Ratios during Walking. Gait Posture, 35, 272-276. https://doi.org/10.1016/j.gaitpost.2011.09.019

[12] Ishikawa, Y., Miyakoshi, N., Kasukawa, Y., Hongo, M. and Shimada, Y. (2013) Spinal Sagittal Contour Affecting Falls: Cut-Off Value of the Lumbar Spine for Falls. Gait Posture, 38, 260-263. https://doi.org/10.1016/j.gaitpost.2012.11.024

[13] Imagama, S., Ito, Z., Wakao, N., Seki, T., Hirano, K., Muramoto, A., et al. (2013) Influence of Spinal Sagittal Alignment, Body Balance, Muscle Strength, and Physical Ability on Falling of Middle-Aged and Elderly Males. European Spine Journal, 22, 1346-1353. https://doi.org/10.1007/s00586-013-2721-9

[14] van der Jagt-Willems, H.C., de Groot, M.H., van Campen, J.P.C.M., Lamoth, C.J.C., and Lems, W.F. (2015) Associations between Vertebral Fractures, Increased thoracic Kyphosis, a Flexed Posture and Falls in Older Adults: A Prospective Cohort Study. BMC Geriatrics, 15, Article No. 34.

https://doi.org/10.1186/s12877-015-0018-Z

[15] Hausdorff, J. M. (2007) Gait Dynamics, Fractals and Falls: Finding Meaning in the Stride-to-Stride Fluctuations of Human Walking. Human Movement Science, 26, 555-589. https://doi.org/10.1016/j.humov.2007.05.003

[16] Hamacher, D., Singh, N.B., Van Dieën, J.H., Heller, M.O. and Taylor, W.R. (2011) Kinematic Measures for Assessing Gait Stability in Elderly Individuals: A Systematic Review. Journal of the Royal Society Interface, 8, 1682-1698. https://doi.org/10.1098/rsif.2011.0416

[17] Gardiner, S., Glogowska, M., Stoddart, C., Pendlebury, S., Lasserson, D. and Jackson, D. (2017) Older PEople's Experiences of Falling and Perceived Risk of Falls in the Community: A Narrative Synthesis of Qualitative Research. International Journal of Older People Nursing, 12, Article No. e12151. https://doi.org/10.1111/opn.12151

[18] Middleton, A., Fritz, S.L. and Lusardi, M. (2015) Walking Speed: The Functional Vital Sign. Journal of Aging and Physical Activity, 23, 314-322.

https://doi.org/10.1123/japa.2013-0236

[19] Motoshige, Y. and Aida, N. (2011) Basic Study on Differences in Static and Dynamic Postural Sway by Type of Footwear. Journal of Japan Society of Nursing and Health Care, 13, 42-49.

[20] Kyrdalen, I.L., Thingstad, P., Sandvik, L. and Ormstad, H. (2019) Associations between Gait Speed and Well-Known Fall Risk Factors among Community-Dwelling Older Adults. Physiotherapy Research International, 24, Article No. e1743. https://doi.org/10.1002/pri.1743

[21] Inoue, T. (2018) Risk Factors for Falls in Terms of Attention during Gait in Community-Dwelling Older Adults. Geriatrics \& Gerontology International, 18, 1444-1586. https://doi.org/10.1111/ggi.13462

[22] Haines, T.P., Bennell, K.M. and Osborne, R.H. (2004) Effectiveness of Targeted Falls Prevention Programme in Subacute Hospital Setting: Randomised Controlled Trial. BMJ Open, 328, Article No. 676. https://doi.org/10.1136/bmj.328.7441.676 